\title{
Cranioplastia externa para a síndrome do trefinado - nota técnica
}

\section{External Cranioplasty for the Syndrome of Trephined - Technical Note}

\author{
Rodrigo Moreira Faleiro ${ }^{1}$ Luiz Alberto Otoni Garcia ${ }^{2}$ Luanna Rocha Vieira Martins ${ }^{3}$ \\ ${ }^{1}$ Chefe do Serviço de Neurocirurgia e Neurologia do Hospital João \\ XXIII; Neurocirurgião do Hospital Felício Rocho, Belo Horizonte, MG, \\ Brasil \\ 2 Residente em Neurocirurgia da Fundação Hospitalar do Estado de \\ Address for correspondence Rodrigo Moreira Faleiro, MD, Rua \\ Caraça, 518, apto 201, Serra, Belo Horizonte, MG, Brasil CEP: 30220- \\ 260 (e-mail: r.m.faleiro@hotmail.com). \\ Minas Gerais, Belo Horizonte, MG, Brasil \\ ${ }^{3}$ Acadêmica de Medicina da Universidade Federal de Minas Gerais, \\ Belo Horizonte, MG, Brasil \\ Arq Bras Neurocir 2015;34:338-341.
}

\section{Resumo \\ Palavras-Chave \\ - craniectomia descompressiva \\ - síndrome do trefinado \\ - cranioplastia externa}

A craniectomia descompressiva (CD) é método cirúrgico amplamente utilizado para tratar a hipertensão intracraniana. Entre as complicações decorrentes da CD, a hidrocefalia é uma das mais frequentes, ocorrendo em até 30\% dos casos. A implantação de derivação liquórica, necessária em alguns casos de hidrocefalia, resulta em agravamento da depressão do couro cabeludo, que ocorre após CD - síndrome do trefinado (ST). A realização de cranioplastia convencional é inviável em algumas situações, como ST com derivação liquórica, que não melhora após ligadura ou aumento de pressão do sistema, ou ST com depressão acentuada do couro cabeludo. Nestes casos, propomos uma nova técnica que foi aplicada em quatro casos e que consiste na confecção de uma prótese temporária com material gessado ou polimetilmetacrilato para se eliminar o efeito da pressão atmosférica. Em todos os casos, houve melhora da ST.

The decompressive craniectomy (DC) is a surgical method widely used to treat intracranial hypertension. Among the complications of $C D$, hydrocephalus is one of the most frequently occurring in up to $30 \%$ of cases. The implantation of CSF shunt, required in some cases of hydrocephalus results in worsening of the scalp depression, which occurs after CD - syndrome of trephined (ST). The performance of conventional cranioplasty is impossible in some situations, such as ST with CSF shunt that does not improve after ligation or increment of system pressure or ST with severe depression of the scalp. In these cases, we propose a new technique that has been applied in four cases and consists in molding a temporary prosthesis with cast material or polymethylmetacrilate to eliminate the effect of atmospheric pressure. In all cases, there was improvement in ST. received

November 10, 2013

accepted

August 7, 2015

published online

October 2, 2015
DOI http://dx.doi.org/

$10.1055 / \mathrm{s}-0035-1564823$. ISSN $0103-5355$.
Copyright $(2015$ by Thieme Publicações License terms

Ltda, Rio de Janeiro, Brazil
(1) $\Theta \circledast$ 


\section{Introdução}

A craniectomia descompressiva (CD) é método cirúrgico amplamente utilizado para tratar a hipertensão intracraniana (HIC) após trauma cranioencefálico (TCE) ou doença cerebrovascular. Consiste em craniectomia e ampliação da dura-máter para se acomodar o cérebro tumefeito.
A CD pode gerar complicações em aproximadamente $50 \%$ dos casos. As complicações mais frequentes são expansão do hematoma ou da contusão (6-58\%); aparecimento de um novo hematoma contralateral ao defeito ósseo (6-28\%); epilepsia (3-22\%); herniação de tecido cerebral através do defeito ósseo (15-51\%); fístula liquórica (2-4\%); infecção (4-16\%); efusão subdural (11-62\%); reabsorção óssea (17\%); hidrocefalia

Tabela 1 Variação da síndrome do trefinado

\begin{tabular}{|l|l|l|l|l|l|l|}
\hline Paciente & Sexo & Idade & Etiologia & Hidrocefalia & DVP & $\begin{array}{l}\text { Síndrome do trefinado - tempo } \\
\text { de surgimento }\end{array}$ \\
\hline 1. NLLF & Masculino & 27 & TCE & Sim & Sim & 1 mês \\
\hline 2. CNL & Masculino & 33 & TCE & Sim & Sim & 4,5 meses \\
\hline 3. PHRA & Masculino & 18 & TCE & Sim & Sim & 3 meses \\
\hline 4. RMSN & Feminino & 40 & HSAE & Sim & Sim & 1 mês \\
\hline
\end{tabular}

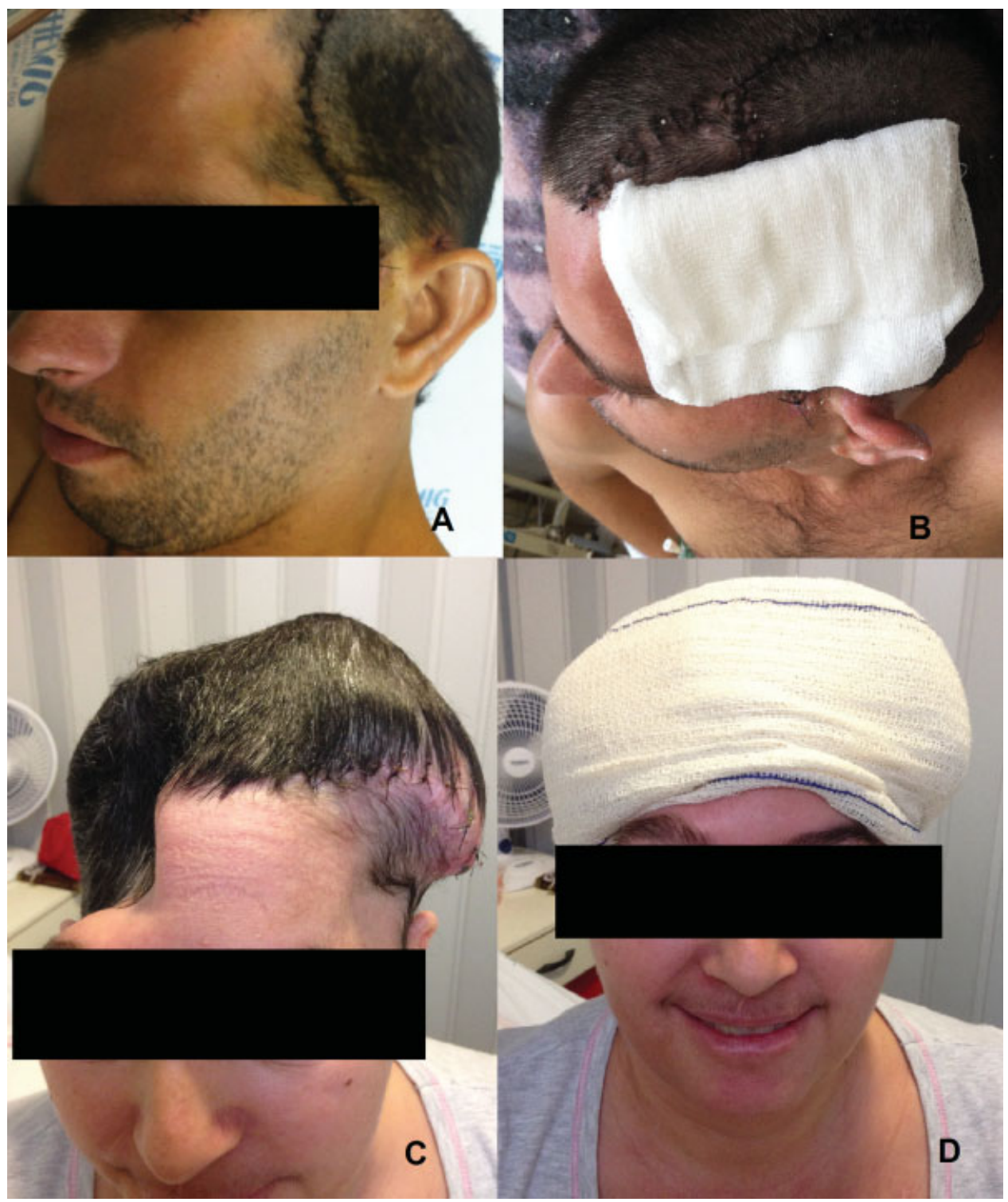

Fig. 1 Prótese externa temporária com material gessado ou com PMMA (40 gramas) utilizada para eliminar efeito da pressão atmosférica. 
(8-29\%) e síndrome do trefinado (1-13\%). As duas últimas ocorrem geralmente após um mês do procedimento. ${ }^{1-6}$

A hidrocefalia é uma complicação relativamente comum após a realização de $\mathrm{CD}$. Sua incidência difere de acordo com o estudo analisado. Quando ocorre, pode ser necessária a inserção de uma derivação liquórica, o que agrava a depressão do couro cabeludo que geralmente ocorre após a CD síndrome do trefinado (ST). Nestes casos, realiza-se cranioplastia no local da falha óssea com o propósito de eliminar o efeito da pressão atmosférica e melhorar o estado neurológico do paciente. ${ }^{4}$

Em nosso serviço, percebeu-se que, em alguns casos, a realização da cranioplastia convencional é inviável em decorrência da presença de ST com derivação liquórica, que não melhora após ligadura ou aumento da pressão do sistema (no caso de válvula regulável), e ST sem derivação liquórica e com depressão profunda do couro cabeludo. Nestes casos, propomos uma nova técnica que consiste na confecção de uma prótese externa com material gessado ou com polimetilmetacrilato (PMMA) para se eliminar o efeito da pressão atmosférica. A seguir, é relatada a série de casos que envolvem as situações descritas, em que optamos pela realização desta técnica, a que denominamos cranioplastia externa.

\section{Relato de Casos}

Trata-se de descrição da técnica (cranioplastia externa) que foi aplicada em quatro casos.
Quanto ao perfil epidemiológico dos pacientes analisados, três eram do sexo masculino, e a faixa etária variava de 18 a 40 anos. Três pacientes eram vítimas de TCE e um possuía hemorragia subaracnóidea decorrente de aneurisma cerebral. Na evolução do quadro, todos os pacientes foram submetidos à $\mathrm{CD}$, cursando com hidrocefalia. Em todos os pacientes foi necessária a implantação de derivação liquórica - derivação ventriculoperitoneal (DVP). A ST foi observada em todos os casos, sendo que seu tempo de aparecimento variou de um a quatro meses e meio. (-Tabela $\mathbf{1}$ ).

Em todos os pacientes, foi confeccionada uma prótese externa temporária com material gessado ou com PMMA (40 gramas), que foi utilizada com o objetivo de eliminar o efeito da pressão atmosférica. Na confecção da prótese, são utilizadas duas compressas para preencher a depressão da pele, facilitando, assim, o molde de gesso ou PMMA. Tomografia computadorizada realizada em até 72 horas evidenciou melhora da ST (-Figs. 1 e $\mathbf{2}$ ). Esta prótese foi mantida até a realização da cranioplastia convencional, com troca diária de curativo. Não houve complicações diretamente relacionadas à técnica ou ao material utilizado.

\section{Discussão}

A CD é uma técnica cirúrgica amplamente utilizada para o tratamento da HIC secundária ao TCE ou a doenças cerebrovasculares. A CD pode resultar em inúmeras complicações, sendo a hidrocefalia uma das mais frequentes, ocorrendo em até $30 \%$ dos casos.

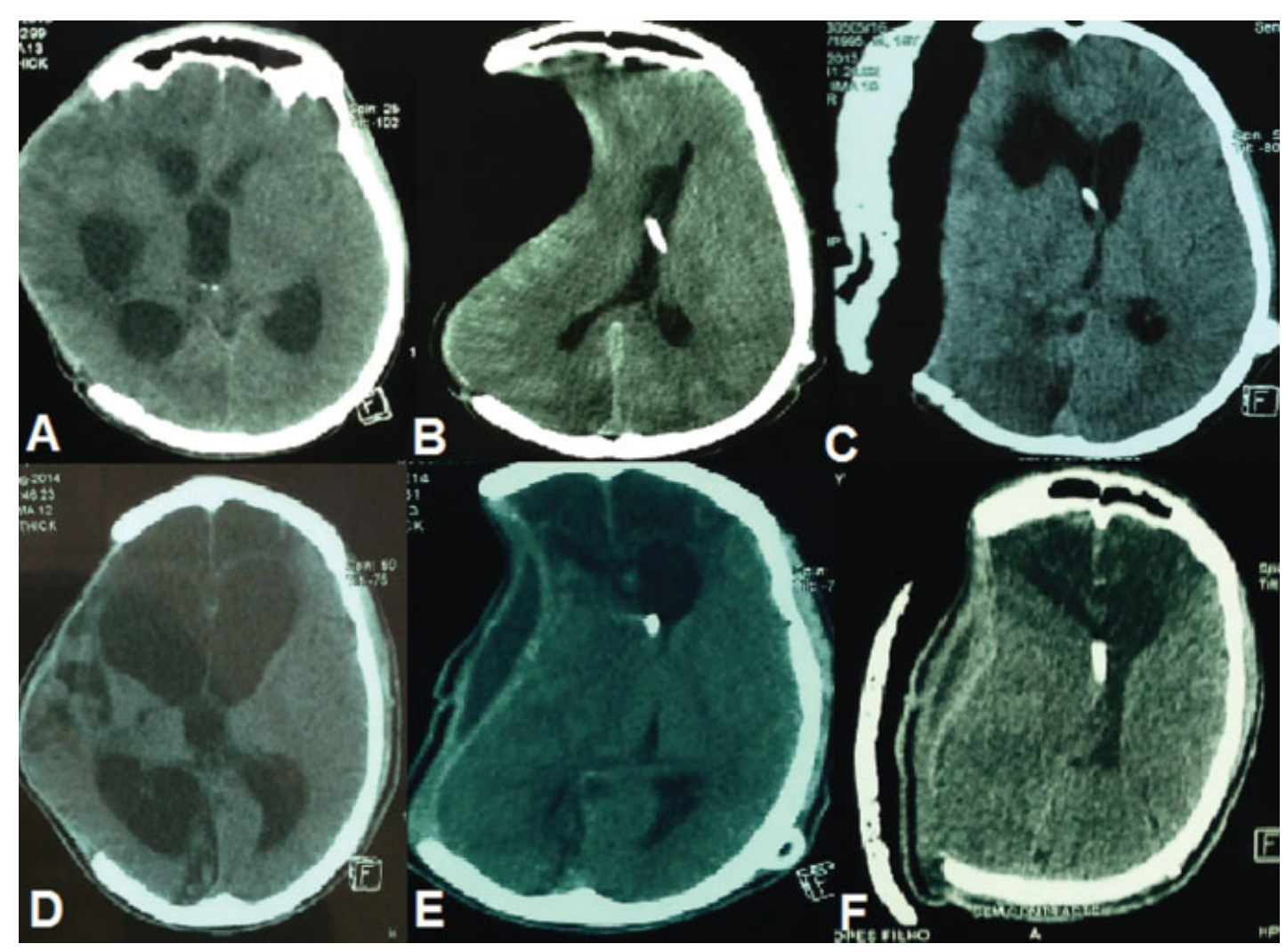

Fig. 2 Tomografia computadorizada realizada em até 72 horas. 
O líquor é um fluido aquoso e incolor que ocupa o espaço subaracnóideo e as cavidades ventriculares e tem como principal função a proteção mecânica do sistema nervoso central. É formado nos plexos corioides e pelo epêndima das paredes ventriculares. O volume total de líquor é de 100 a 150 $\mathrm{cm}^{3}$, renovando-se completamente a cada 8 horas. É absorvido nas granulações aracnóideas que se projetam no interior dos seios da dura-máter, sendo predominante no seio sagital superior. Para que esta absorção ocorra, deve haver uma diferença pressórica entre o espaço subaracnóideo e os seios da dura-máter. A pressão do líquor no espaço subaracnóideo é em torno de $0-10 \mathrm{mmHg}$ no adulto, e a pressão dos seios durais é negativa, refletindo a pressão da cavidade torácica. 6,7

Segundo Bateman et al, estados de hiperemia encefálica cursam com aumento da pressão venosa, ou seja, da pressão nos seios da dura-máter. ${ }^{8}$ Como a CD é realizada em casos de brain swelling, ou seja, hiperemia encefálica, e sabidamente causa uma queda abrupta da pressão subaracnóidea, temos a possível explicação da formação da hidrocefalia após realização de CD.

Para tratar a hidrocefalia, deve ser implantada uma derivação liquórica, que pode ser interna ou externa. Este procedimento causa um aumento da depressão no local da craniectomia como resultado do gradiente de pressão atmosférica - ST. Dessa forma, deve-se tentar aliviar o efeito da pressão atmosférica no local da craniectomia, o que pode ser feito com cranioplastia. Contudo, em alguns casos, como já relatado, a realização da cranioplastia convencional é inviável em decorrência da presença de ST com derivação liquórica, que não melhora após ligadura do sistema ou aumento de pressão (no caso de válvula regulável), e ST sem derivação liquórica e com depressão profunda do couro cabeludo. Outra situação seria a presença de ST com extensa necrose de pele. Para diminuir o grau de depressão, optou-se, em nosso serviço, pela realização de cranioplastia externa com material gessado ou polimetilmetacrilato, com bons resultados.

\section{Referências}

1 Yang XF, Wen L, Shen F, et al. Surgical complications secondary to decompressive craniectomy in patients with a head injury: a series of 108 consecutive cases. Acta Neurochir (Wien) 2008; 150(12):1241-1247, discussion 1248

2 Flint AC, Manley GT, Gean AD, Hemphill JC III, Rosenthal G. Post-operative expansion of hemorrhagic contusions after unilateral decompressive hemicraniectomy in severe traumatic brain injury. J Neurotrauma 2008;25(5): 503-512

3 Ban SP, Son YJ, Yang HJ, Chung YS, Lee SH, Han DH. Analysis of complications following decompressive craniectomy for traumatic brain injury. J Korean Neurosurg Soc 2010;48(3): 244-250

4 Faleiro RM, Faleiro LC, Caetano E, et al. Decompressive craniotomy: prognostic factors and complications in 89 patients. Arq Neuropsiquiatr 2008;66(2B):369-373

5 Honeybul S, Ho KM. Long-term complications of decompressive craniectomy for head injury. J Neurotrauma 2011;28(6): 929-935

6 Honeybul S. Complications of decompressive craniectomy for head injury. J Clin Neurosci 2010;17(4):430-435

7 Machado A, Haertel LM. Neuroanatomia funcional. 3. Ed. São Paulo: Atheneu; 2006

8 Bateman G. Hyperemic hydrocephalus: a new form of childhood hydrocephalus analogous to hyperemic intracranial hypertension in adults. J Neurosurg Pediatr 2010;5(1):20-26 